\title{
Research and Implementation of smooth transition strategies --- IPv6 tunnel technology
}

\author{
YaFang Lou ${ }^{1, a}$ YongBing $\mathrm{Xu}^{2, \mathrm{~b}}$ \\ ${ }^{1,2}$ Department of Computer Science and Technology \\ ZhuHai College Of JiLin University , 519041 \\ ZhuHai,China \\ aluckylou@sohu.com, ${ }^{\mathrm{b}}$ fenjiexyb@163.com
}

\author{
ZhiJun Yuan ${ }^{3, c}$ \\ Pillar Resource Services Inc 4155-84th Ave ,T6B $2 Z 3$ \\ Edmonton, Alberta, Canada \\ 'cyy_zjy@yahoo.ca
}

\begin{abstract}
IPv6 has a massive address ,multicast ,neighbor discovery, auto-configuration, service quality, removable and many other new features, as the core technology of the next generation IP( Internet Protocol). IPv4 to IPv6 transition is imperative. This paper discusses how to achieve a smooth transition from IPv4 to IPv6 and researches deeply IPv6 tunneling technologies and IPv6 6to4 automatic tunneling technologies in the Windows circumstances.
\end{abstract}

Keywords- IPv4; IPv6; Tunnel; Windows

\section{INTRODUCTION}

With the rapid developing of Internet technology, the limited address space IPv4 defined in the Internet is running out and the lack of address space will become the main factors to hinder the internet further developing . IPv6, a new version of Internet protocol is proposed to redefine address space and then to replace the IPv4 protocol. As the core technology of the next generation Internet protocol,IPv6 has many new features,such as massive addresses, multicast, neighbor discovery, auto-configuration, service quality, removable and so on. It is imperative that the current IPv4 is migrated to IPv6. IETF has established a special working team to study the transition from IPv4 to IPv6 and has proposed a number of strategies. Among of them, there are three main technologies: IPv4/IPv6 dualstack, address / protocol conversion and IPv6-based tunnel technology This paper mainly studies the IPv6 tunnel mechanism and implementation of IPv6 tunnel technology in the Windows environment.

\section{IPV4 TO IPV6 TRANSITION STRATEGY}

Currently, a large number of networks is IPv4. With the deployment of IPv6, the transition between the IPv4 network today and the IPv6 network in the future will not be a short process during both protocols coexistence. IPv4 to IPv6 transition is broadly divided into three stages:

The initial stage: IPv4 protocol dominates networks absolutely. The IPv6 isolated islands are dotted in IPv4 ocean, these Ipv6 islands are connected by Ipv4 ocean, with the tunneling technology.

Coexistence phases: With the deployment of the IPv6 network, IPv6 networks are got large-scale applications and certain IPV6 backbone networks are formed. The business in Ipv6 platform will continue to be increased. The different
IPv6 networks are connected mutually with IPv4 networks and the communication between IPv4 and IPv6 host is implemented by Ipv4 networks[1]. In this stage, not only the dual-stack and tunneling but also the network protocol conversion technology will be used.

Dominant stage: IPv6 networks and hosts dominate. Later, when IPv6 is developed to cover all the backbone networks, IPv4 network became islands. Tunneling technology is main deployment.

IPv6 network deployment process, as shown in Figure 1.

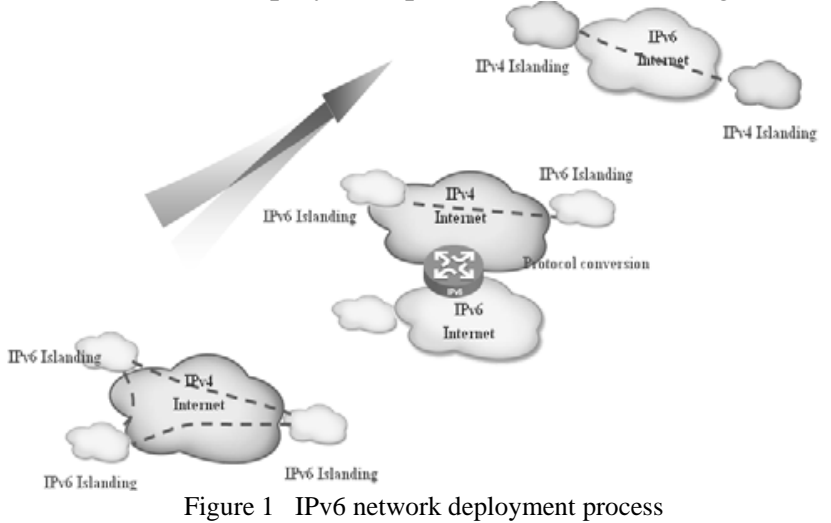

\section{IPv6 TUNNEL TECHNOLOGY}

Tunnel technology is that a kind of protocol is encapsulated to another protocol. Tunnel only requires the two tunnel end's equipments (i.e. two protocol boundary point of intersection of the two protocol) support the two protocols. The IPv6 passing through IPv4 Tunnel technology[2], offers using the existing IPv4 network to provide connectivity for the independent IPv6 network, the IPv6 message is encapsulated to IPv4 message to pass through the IPv4 network, realizing the transparent transmission for IPv6 message.The IPv6 network edge device, after receiving the IPv6 message form IPv6 network ,encapsulates the IPv6 message to the IPv4 message to become a IPv4 message. Then the new IPv4 message , after having a transmission in the IPv4 network to a destination IPv6 network edge device, decapitates to remove the external IPv4 head ,to restore the original IPv6 message, and then have IPv6 retransmission

Tunnel technology has the advantage, that not all the equipment is upgraded to dual stack, but only requires the 
IPv4 /IPv6 network edge device having the function of dual stack and tunnel .Except the edge node, the rest nodes need no supporting the dual stack protocol. The structure as shown in Figure 2.

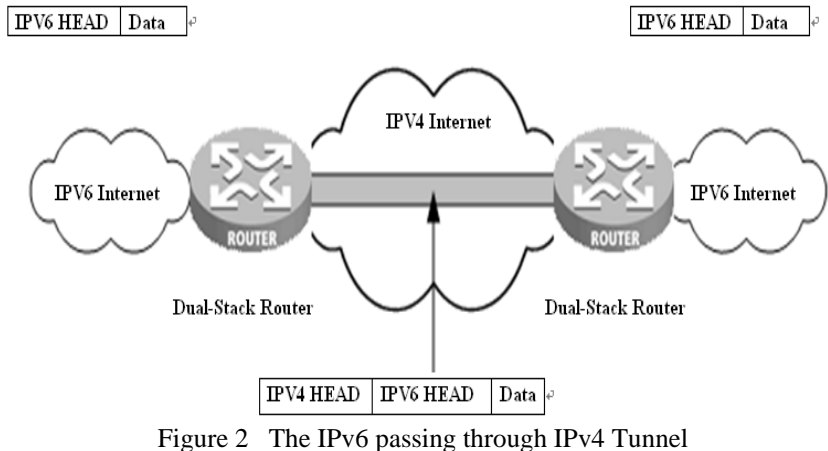

The mainly tunnel technologies in the IPv6 passing through the IPv4 network[3] :

(1) IPv6 manually configured tunnel

(2) 6to4 automatic tunnel

(3) ISATAP automatic tunnel

(4) IPv6 over IPv4 GRE tunnel

(5) $6 \mathrm{PE}$

\section{A. IPv6 manually configured tunnel}

The IPv6 manually configured tunnel's source address and destination address are manually specified, providing a P2P connection .IPv6 manually configured tunnel not only can be established between two boundary routers to provide the IPv6 network separated by the IPv4 network with a stable connection ,but also can be established between the terminal system and the boundary router to provide terminal system a connection for visiting the IPv6 network. The tunnel endpoint device must supports the IPv6/IPv4 dual stack. Other devices need only implement a single stack[4].

The IPv6 manually configured tunnel requires that it should be on the device to manually configure the tunnel source address and destination address. If there is a edge device wants to establish manual tunnel towards other several devices ,the edge device should be configured the same number of the tunnels. Thus, the IPv6 manually configured tunnel is normally used to between two edge routers, offer a connection between 2 IPv6 networks.

The manually configured tunnel as a virtual interface(A ) existed on the device, after receiving a IPv6 message from a IPv6 network, looks for the IPv6 forwarding table according to the destination address of the IPv6 message. If the message is of retransmission from the virtual interface(A ), then it will be encapsulated according to the source port's IPv4 address and destination port's IPv4 address which are configured by the tunnel interface . After being encapsulated ,the message becomes into a IPv4 message to be processed by the IPv4 protocol stack. The message via IPv4 network retransmits to the tunnel's terminal point. After receiving a tunneling protocol message, the tunnel's terminal point has a tunneling decapsulation and sends the message decapitated to IPv6 protocol stack to be processed. It is not allowed to , on one device ,have two IPv6 manually tunnels having the both same source address and destination address.

\section{B. 6 to4 automatic tunnel}

6to4 tunnel is a kind of automatic tunnel [5],too, and it also uses the IPv4 address which is embedded in IPv6 address to be established . 6to4 automatic tunnel has the support for Router to Router ,Host to Router ,Router to Host , and Host to Host .

The 6to4 automatic tunnel enables the isolated IPv6 networks to connect via the IPv4 network

with each other . 6to4 automatic tunnel is realized by the tunneling virtual interface . 6to4 tunneling entrance's IPv4 address is manually specified, and the tunnel's destination address is decided by the message which pass through the tunnel to retransmit. If the IPv6 message's destination address is not classified as a 6to 4 address , the IPv4 address which is extracted from the message's destination address would be the tunnel's destination address, and if not ,besides, the next hop's address is classified as a 6 to 4 address, the IPv4 address which is extracted from the next hop's address would be the tunnel's destination address. The latter is also called 6to4 relaying.

A IPv6 message ,after arriving in the edge router ,according to the message's IPv6 destination address to look for the forwarding table, if the outputting interface is the 6to4 automatic tunnel's Tunnel virtual interface and the message's destination address or the next hop's address is a 6 to 4 address , the IPv4 address which is extracted from the 6 to 4 address would be the tunneling message's destination address and the tunneling message's source address is on the Tunnel interface to be configured.

One IPv4 address is only used to one 6to 4 tunneling source address ,if a edge router has many 6to 4 networks using the same IPv4 address as their respective network local address ,the edge router will use the 6to4 address's SLAID to distinguish them ,but they share a tunnel.

\section{ISATAP automatic tunnel}

ISATAP(Intra-Site Automatic Tunnel Addressing Protocol) is another kind of IPv6 automatic tunneling technology. As similar to the 6to4 address, ISATAP has the IPv4 address embedded in it. Its tunneling encapsulation also works according to the IPv4 address above. Just the ISATAP address format differs on the 6to 4 address format. 6 to 4 uses the IPv4 address as its network ID while ISATAP uses IPv4 address as its interface ID.

If the IPv4 address is globally unique, the bit u should be 1 , if not ,the bit $u$ should be 0 . The bit $g$ is the sign of IEEE group/individual . The format of ISATAP addressing interface ID looks like 00-00-5E-FE plus IPv4 address. 5EFE was distributed by IANA. 
Because the ISATAP is expressed through the interface ID ,the ISATAP address has the various formats ,like global unicast ,site unicast, multicast . The first 64 bits send the requirement to the ISATAP router to get data for itself, and it has the automatic address configuration. The ND protocol can be running between the two end devices of the ISATAP tunnel.

ISATAP tunnel regards the IPv4 network as a nonbroadcast , point-to-multipoint link(NBMA ).

The ISATAP's transition mechanism allows deploying the IPv6 ,which is implemented inside the existing IPv4 network .This technology is simple and it has quite good expansibility, which can be used for local site transition. ISATAP supports the IPv6 site local router globally IPv6 routing region and the automatic IPv6 tunnel. ISATAP can also combined with NAT ,then to use the globally unique IPv4 address inside the site.

\section{IPv6 over IPv4 GRE tunnel}

IPv6 over IPv4 GRE tunnel uses the standard GRE tunnel technology to provide a P2P connecting service ,which needs manually specifying the tunnel's endpoint address. GRE tunnel itself doesn't limit the passenger protocol and transmission protocol, but in the implement of Comware, the GRE tunneling transmission protocol is fixed while the passenger protocol can be every protocol which is allowed by itself(it could be the Pv4 ,IPv6 ,OSI ,MPLS ,etc ).

\section{E. 6PE tunnel}

If the service provider wants to realize a IPv6 network, for the situation that the network crisis is based on the IPv4, he can structure the IP tunnels between the edge routers which can support the IPv6 protocol, the IP tunnels can be the P2P connections which can support the IPv6 protocol. The IPv6 packet, which switch among these above edge routers ,can be encapsulated to IP packet to transparently transmit in the backbone network. When it comes to the large-scale network, the Scalability of these schemes is not so good; so the MPLS technology supply another choice :transmit the IPv6 datagram in the IPv4 backbone which has the starting MPLS.

Provider's Edge Router (6PE),provides a solution for the telescopic IPv6 early deployment.

ISP uses the existing IPv4 backbone networks to provide access to the IPv6 networks dispersing the users. ISP's main idea is that the user's IPv6 routing information is transformed into the IPv6 routing information with labels, and then spreads via the IBGP (Internal Border Gateway Protocol ) conversation to the ISP's IPv4 backbone networks .When the IPv6 message retransmits, the flow entering the backbone network tunnel will be labeled firstly .The tunnel may be the GRE tunnel or the MPLS LSP ,etc.

\section{Tunneling technology implementing}

After discussing the tunnel, we built an experimental environment to establish IPv6 tunnel. The tunnel type: 6to4 tunnel. Both of IPv6 hosts act not only as the two ends of the tunnel, but also as the source and destination sites of transmitting IPV6 datagram through the IPv4 network. Among of R2, R3 and R5, Ipv4 network is established and is configured the IP address and start routing[6]. Both R1 and R5 are IPv6 addresses. They communicate with each other through the IPV4 network. Topology shown in Figure 3

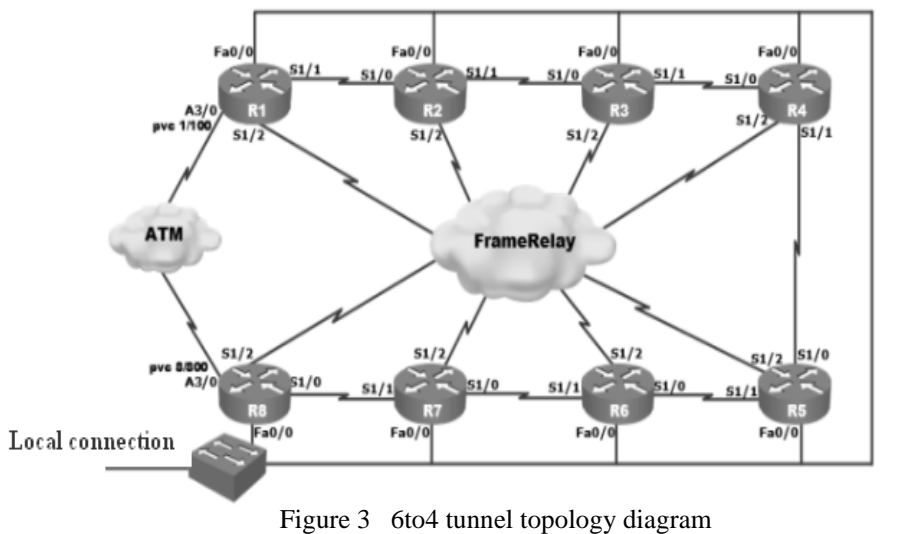

6to4 tunnel configuration command:

//First : establish IPv4 network among R2, R3, R4

R2(CONFIG)\#interface s1/1

R2(CONFIG -if)\#IP address 2.2.2.1 255.255.255.0

R2(CONFIG -if)\#no shut

R2(CONFIG -if)\#

R3(CONFIG)\#interface s1/0

R3(CONFIG -if)\#IP address 2.2.2.2 255.255.255.0

R3(CONFIG -if)\#no shut

R3(CONFIG -if)\#no shutdown

R3(CONFIG -if)\#exit

R3(CONFIG)\#inter

R3(CONFIG)\#interface s1/1

R3(CONFIG -if)\#IP address 3.3.3.2 255.255.255.0

R3(CONFIG -if)\#no shut

R3(CONFIG -if)\#

R4(CONFIG)\#interface s1/0

R4(CONFIG -if)\#IP address 3.3.3.1 255.255.255.0

R4(CONFIG -if)\#no shut

R4(CONFIG -if)\#no shut

R4(CONFIG -if)\#no shutdown

//Enable static routing in the R2 and R4, enable the network connectivity

R2 (CONFIG) \# IP route 3.3.3.0 255.255.255.0 2.2.2.2

R4 (CONFIG) \# IP route 2.2.2.0 255.255.255.0 3.3.3.2

//To get through the tunnel in R2, R4

R2 (CONFIG) \# interface tunnel 0

R2 (CONFIG -if) \# no IP address

R2 (CONFIG -if) \# ipv6 address 2001:250:803:1 :: 1/64

R2 (CONFIG -if) \# tunnel source 2.2.2.1 (R2 e0 / 1)

R2 (CONFIG -if) \# tunnel destination 3.3.3.1 (R4 e0 / 0)

R2 (CONFIG -if) \# tunnel mode ipv6ip

R4 (CONFIG) \# interface tunnel 1

R4 (CONFIG -if) \# no IP address 
R4 (CONFIG -if) \# ipv6 address 2001:250:803:1 :: 2/64

R4 (CONFIG -if) \# tunnel source 3.3.3.1 (R4 e0 / 0)

R4 (CONFIG -if) \# tunnel destination 2.2.2.1 (R2 e0 / 1)

R4 (CONFIG -if) \# tunnel mode ipv6ip

//Configure the IPv6 addresses in R1, R2,R4,R5

R1 (CONFIG) \# ipv6 unicast-routing

R1 (CONFIG) \# interface s1 / 1

R1 (CONFIG -if) \# ipv6 address 2001:250:803:11 :: 1/64

R1 (CONFIG -if) \# no shut

R2 (CONFIG) \# ipv6 unicast-routing

R2 (CONFIG) \# interface s1 / 0

R2 (CONFIG -if) \# ipv6 address 2001:250:803:11 :: 2/64

R2 (CONFIG -if) \# no shut

R4 (CONFIG) \# ipv6 unicast-routing

R4 (CONFIG) \# interface s1 / 1

R4 (CONFIG -if) \# ipv6 address 2001:250:803:12 :: 1/64

R4 (CONFIG -if) \# no shut

R5 (CONFIG) \# ipv6 unicast-routing

R5 (CONFIG) \# interface s1 / 0

R5 (CONFIG -if) \# ipv6 address 2001:250:803:12 :: 2/64

R5 (CONFIG -if) \# no shut

//Setup static routes in R1, R2, R4, R5

R1 (config) \# ipv6 route 2001:250:803:1 :: / 64

2001:250:803:11:: 2

R1 (config) \# ipv6 route 2001:250:803:12 :: / 64

2001:250:803:11:: 2

R2 (config) \# ipv6 route 2001:250:803:12 :: / 64

2001:250:803:1 :: 2

R4 (config) \# ipv6 route 2001:250:803:11 :: / 64

2001:250:803:1 :: 1
R5 (config) \# ipv6 route 2001:250:803:1 :: / 64

2001:250:803:12:: 1

R5 (config) \# ipv6 route 2001:250:803:11 :: / 64

2001:250:803:12:: 1

//Configured.

By testing the tunnels between R1 and R5, R2 tunnel R4, 6 to4 tunnel has been connected

\section{CONCLUSION}

This paper discusses the IPv6 network deployment process and its three transition strategies and researches the principles of IPv6 tunneling strategies and the related technical problems .It validate and implements the IPv6 6to4 tunnel strategy by simulation. IPv6 is considered as the core technology of the next generation internet. It is very advantageous for us to plan the future development of the network application, Understanding and researching IPv6 principle, IPv6 tunnels and the related technologies, it is very advantageous for us to plan the future development of the network application.

\section{REFERENCES}

[1] Niall Murphy, David Malone (Author) IPv6 Network Administration [M] O'Reilly Media, Inc. (March 2, 2005)

[2] Joseph Davies. Understanding IPv6Second Edition [M]. Microsoft Press. 2008

[3] http:/ / tb. 6test. Edi. Cn / win xp conf. Txt 2002

[4] IETFRFC 2766 2000, Network Address Translation protocol Translation (NATPT) [S]

[5] Rob Coltun, Dennis F erg uson, Jo hn M o y. O SPF for IPv6 [S]. RF C2740, 1999.

[6] Desmeules R. Cisco IPv6 Network technology [M]. Beijing: People's Posts and Telecommunications Press, 2004 